\title{
GENERAL POSITION OF POINTS ON A RATIONAL RULED SURFACE
}

\author{
ALBERTO ALZATI AND ALFONSO TORTORA
}

\begin{abstract}
In this note we introduce a definition of general position for distinct points on a rational ruled surface and we discuss some related properties having in mind very ampleness criteria for rank 2 vector bundles.
\end{abstract}

\section{INTRODUCTION}

The notion of points in general position on a plane is well known, with some different definitions, and very useful in many contexts. For instance, in $[\mathrm{A}-\mathrm{T}]$ the authors studied rank 2 vector bundles $E$ whose sections have a zero locus with this property showing that there are very ampleness criteria based upon it. However an analogous notion for points on other surfaces was not exploited in the literature, not even for rational surfaces. The notion of separator for a set $\Delta$ of points on a surface (see [B-C]) is somehow related to "generic position" of points of $\Delta$ but it is not the natural generalization of the usual definition we pursue.

If $S$ is a rational surface whose minimal model is $\mathbb{P}^{2}$ and $\sigma: S \rightarrow \mathbb{P}^{2}$ is the blowing up of $\mathbb{P}^{2}$ at some points $Q_{1}, \ldots, Q_{t}$, (not necessarily distinct), there is a natural definition of general position for a set of distinct points $P_{1}, \ldots, P_{r} \in S$, i.e. $\left\{P_{1}, \ldots, P_{r}\right\}$ are in general position on $S$ if and only if $\left\{\sigma\left(P_{1}\right), \ldots, \sigma\left(P_{r}\right), Q_{1}, \ldots, Q_{t}\right\}$ are in general position on $\mathbb{P}^{2}$ (see for instance $[\mathrm{A}-\mathrm{B}]$ when $t=1$ ). However in this paper we consider rational ruled surfaces $S=\mathbb{F}_{e}$ where different definitions of "points in general position" are possible. We will choose the most natural one, according to our opinion (see Definition 1). Unfortunately, the relation between this property and the very ampleness of rank 2 vector bundles whose generic sections have zero locus in general position (see $\S 4$ and $\S 5$ ) is not very rewarding. In those cases where the relation should help, it turns out that a weaker property than general position is enough: namely that the set of points is disjoint from the unique section $C_{0}$ of $\mathbb{F}_{e}$ (see Corollary 3 and Corollary 4 ).

In $\S 2$ we fix notation and recall some known facts. In $\S 3$ we consider sets of points lying on a smooth section of $S$. In $\S 4$ and in $\S 5$ we show that, although the above mentioned relation is not as fruitful as one may hope, we will succeed in proving the very ampleness of many vector bundles $E$ over $S$ (see for instance Theorems 3, 4 and subsequent Corollaries) with related techniques.

Date: September 5th 2017.

2010 Mathematics Subject Classification. Primary 14J60; Secondary 14J26.

Key words and phrases. rank 2 vector bundles, general position, very ampleness.

This work is within the framework of the national research project "Geometry on Algebraic Varieties" Cofin 2010 of MIUR.. 


\section{Notation AND BaCkground material}

$\mathbb{P}^{N}:=$ projective space of dimension $N$ over $\mathbb{C}$

$\sim:=$ linear equivalence of divisors

$\mathbb{F}_{e}:=$ rational ruled surface of invariant $e \geq 0$ i.e. $\mathbb{F}_{e}=\mathbb{P}\left(\mathcal{O}_{\mathbb{P}^{1}}(-e) \oplus \mathcal{O}_{\mathbb{P}^{1}}\right)$

$C_{0}:=$ tautogical line bundle of $\mathbb{F}_{e}$

$f:=$ numerical class of a fibre of $\mathbb{F}_{e}$

$\operatorname{Pic}\left(\mathbb{F}_{e}\right)=\operatorname{Num}\left(\mathbb{F}_{e}\right)=\left\langle C_{0}, f\right\rangle$

$E:=$ rank 2 vector bundle over a surface $\mathbb{F}_{e}$

$s:=$ section of $E$, element of $H^{0}\left(\mathbb{F}_{e}, E\right)$

$(s)_{0}:=$ zero locus of $s$

$\sharp(A):=$ cardinality of a set $A$ of points

$c_{1}(E):=$ first Chern class of $E$

$c_{2}(E):=$ second Chern class of $E$, identified with an integer

$X:=\mathbb{P}(E)$ projectivization of $E, X$ is a smooth 3-fold

$\pi:=$ natural projection $X \rightarrow \mathbb{F}_{e}$

$T:=$ tautological line bundle of $X$, i.e. $\mathcal{O}_{\mathbb{P}(E)}(1)$

$\left|\mathcal{O}_{V}(D)\right|$ or briefly $|D|:=$ linear system given by the effective divisors on a smooth algebraic variety $V$, linearly equivalent to $D$; a linear system is a projective space of dimension $h^{0}(V, D)-1$

$\mathcal{I}_{Y}:=$ ideal sheaf of a subscheme $Y$ of $V$

$\mathcal{I}_{Y}(D):=\mathcal{I}_{Y} \otimes \mathcal{O}_{V}(D)$

$E(D):=E \otimes \mathcal{O}_{V}(D)$

$K_{V}:=$ canonical divisor of a smooth algebraic variety $V$

$\left\langle Y_{1}, \ldots, Y_{q}\right\rangle:=$ linear span of $q$ linear subspaces in a vector space or linear span of $q$ projective subschemes in a projective space

$A \longleftrightarrow B:=$ there is a bijection between the sets $A$ and $B$.

To avoid too many particular cases, throughout this paper we will always assume $e>0$. When $e=0, \mathbb{F}_{0} \simeq \mathbb{P}^{1} \times \mathbb{P}^{1}$ and there are specific criteria for the very ampleness of vector bundles over Segre products.

Let $\Delta$ be a set of $\delta \geq 1$ distinct points on a rational ruled surface $\mathbb{F}_{e}$ embedded as a scroll in some projective space. To give a suitable definition of general position for $\Delta$ we recall that the surface is ruled in lines, so that sets of points having two or more elements on the same line would be not in general position. Hence, to define the property, we could use only smooth sections of $\mathbb{F}_{e}$, i.e. general curves belonging to a linear system $\left|C_{0}+x f\right|$ for some $x \geq e$. However it is useful to consider also the cases $x \in[0, e)$. Recall that $\left|C_{0}+x f\right|=C_{0}+|x f|$ for $x \in[0, e)$, i.e every element of $\left|C_{0}+x f\right|$ is the union of the fixed section $C_{0}$ and $x$ fibres. The above facts are the motivation of the following definition.

Definition 1. Let $\Delta$ be a set of $\delta \geq 1$ distinct points on a rational ruled surface $\mathbb{F}_{e}$. For any integer $x \geq 0$, let us call $\varphi_{\Delta}(x):=h^{0}\left(\mathbb{F}_{e}, \mathcal{I}_{\Delta}\left(C_{0}+x f\right)\right.$ ) (or simply $\varphi(x)$ when no confusion can arise). We say that $\Delta$ is in general position if, for any $C_{0}+x f \in \operatorname{Pic}\left(\mathbb{F}_{e}\right)$ with $x \geq 0$, we have $\varphi_{\Delta}(x)=\max \left\{h^{0}\left(\mathbb{F}_{e}, C_{0}+x f\right)-\delta, 0\right\}$.

Remark 1. We can calculate $h^{0}\left(\mathbb{F}_{e}, C_{0}+x f\right)$ in any case, so that there is an alternative definition for the general position of $\Delta$.

If $x \in[0, e)$ it must be:

$\varphi_{\Delta}(x)=x+1-\delta$ if $x>\delta-1$

$\varphi_{\Delta}(x)=0$ if $x \leq \delta-1$. 
If $x \geq e$ it must be:

$\varphi_{\Delta}(x)=2 x+2-e-\delta$ if $x>\frac{e+\delta}{2}-1$

$\varphi_{\Delta}(x)=0$ if $x \leq \frac{e+\delta}{2}-1$.

Sometimes we will call "expected value of $\varphi(x)$ " the value satisfying Definition 1. Of course we could give an analogous definition of general position also for 0dimensional subschemes of the surface, however such a different definition would not introduce a substantial improvement in our analysis and we will avoid it.

We recall the following proposition.

Proposition 1. For any rank 2 vector bundle $E$ over $\mathbb{F}_{e}$ there exist two line bundles $L$ and $M$, a 0 -dimensional subscheme $Z$ of $\mathbb{F}_{e}$ and (at least) an exact sequence of sheaves of $\mathbb{F}_{e}$ such that:

$$
0 \rightarrow L \rightarrow E \rightarrow \mathcal{I}_{Z}(M) \rightarrow 0 \quad(\epsilon)
$$

Proof. The existence of $(\epsilon)$ follows from $[\mathrm{F}]$ prop. 5 page 33 and lemma 11 page 92.

Recall that, for a vector bundle sitting in an exact sequence as $(\epsilon)$, we have that $c_{1}(E) \sim L+M$ and $c_{2}(E)=L M+z$, where $z:=\operatorname{length}(Z)$.

Until $\S 5$, in this paper we will consider only rank 2 vector bundles $E$ over $\mathbb{F}_{e}$ sitting in an exact sequence as $(\epsilon)$ with $L \sim C_{0}+\alpha f$ and $M \sim C_{0}+\beta f$. We will show that even in this simple case it is not possible to get a useful relation between the general position of $(s)_{0}$ and the very ampleness of $E$. Recall that the very ampleness of $E$ is equivalent to the existence of the corresponding threefold $X=\mathbb{P}(E)$ in some projective space (embedded by $|T|$ ) and such threefolds are important in the classification theorems of projective threefolds of low degree (see [F-L1], [F-L2], [B-B] and also [I1] and [I2]). We have:

Proposition 2. Let $E$ be a rank 2 vector bundle over $\mathbb{F}_{e}$ sitting in an exact sequence as $(\epsilon)$ with $L \sim C_{0}+\alpha f$ and $M \sim C_{0}+\beta f$ and let $V \subseteq H^{0}\left(\mathbb{F}_{e}, E\right)$ be any linear subspace of sections of $E$. Then:

i) if $E$ is very ample, $Z=\emptyset$ and $\beta>e$;

ii) if $Z=\emptyset$ any generic section $s$ of $E$ determines a divisor $\Gamma_{s} \in|M|$ and $(s)_{0} \subset$ $\Gamma_{s}$, if $E$ is generated by global sections $(s)_{0}$ is a set of $L M=-e+\alpha+\beta$ distinct points belonging to $\Gamma_{s}$;

iii) if $Z=\emptyset, \beta>e$ and $\operatorname{dim}\left[\operatorname{Im}\left(V \rightarrow H^{0}\left(\mathbb{F}_{e}, M\right)\right)\right]>\beta+1$ then $\Gamma_{s}$ is a smooth rational curve for any generic $s \in V$.

Proof. $i$ ) Assume that $Z \neq \emptyset$, let $P$ be a point of $Z$ and let $f_{P}$ be the fibre passing through $P$. If we restrict $(\epsilon)$ to $f_{p}$ we get: $E_{\mid f_{P}} \rightarrow \mathcal{O}_{\mathbb{P}^{1}}(1-\varepsilon) \rightarrow 0$ where $\varepsilon \geq 1$ is the length of $Z \cap f_{P}$. As $E$ is very ample $\mathcal{O}_{\mathbb{P}^{1}}(1-\varepsilon)$ must be ample, hence $1-\varepsilon>0$, contradiction. When $E$ is very ample and $Z=\emptyset$ then $M$ is ample, hence $\beta>e$.

ii) Let us consider $(\epsilon)$ tensorized by $-L$ :

$$
0 \rightarrow \mathcal{O}_{\mathbb{F}_{e}} \rightarrow E(-L) \rightarrow M-L \rightarrow 0
$$

and let $\mathcal{U}$ be a suitable open subset of $\mathbb{F}_{e}$ where both $E$ and $E(-L)$ are trivial. Let $\sigma=\left(\sigma_{1}, \sigma_{2}\right)$ be a local description in $\mathcal{U}$ of the section of $E(-L)$ given by $0 \rightarrow \mathcal{O}_{\mathbb{F}_{e}} \rightarrow E(-L)$. Locally, the linear map $H^{0}\left(\mathbb{F}_{e}, E\right) \rightarrow H^{0}\left(\mathbb{F}_{e}, M\right)$ acts as follows: $\left(g_{1}, g_{2}\right) \rightarrow \sigma_{1} g_{2}-\sigma_{2} g_{1}$ (see $[\mathrm{G}-\mathrm{H}]$ pag 726 ) for any section $s$ having local description $\left(g_{1}, g_{2}\right)$. Obviously $\gamma_{s}:=\sigma_{1} g_{2}-\sigma_{2} g_{1}=0$ is the local description of a 
divisor belonging to $|M|$. Of course $(s)_{0} \subset \Gamma_{s}$. If $E$ is generated by global sections, the zero locus of a generic section of $E$ is a set of $c_{2}(E)=L M$ distinct points.

iii) As $\beta>e$, the only singular curves in $|M|$ belong to subspaces of the type $C_{0}+\beta^{\prime} f+\left|\left(\beta-\beta^{\prime}\right) f\right|$ where $C_{0}+\beta^{\prime} f$ is a fixed curve and $\left|\left(\beta-\beta^{\prime}\right) f\right|$ is the linear system of $\beta-\beta^{\prime}$ fibres (see for instance $[\mathrm{A}-\mathrm{B}]$, Lemma 3.4). The maximal dimension of such subspaces is $\beta+1$ (when $\beta^{\prime}=0$ ), hence, in our assumptions, not every $\Gamma_{s} \in \operatorname{Im}\left(V \rightarrow H^{0}\left(\mathbb{F}_{e}, M\right)\right)$ can be a singular curve, therefore the generic one is smooth.

\section{Points on Smooth Rational CURVes}

Proposition 2 shows that, for our aim, it is crucial to have informations about the general position of points belonging to smooth rational sections. To this end it is is useful the following more general

Proposition 3. Let $\Delta$ be a set of $\delta \geq 1$ distinct points on a rational ruled surface $\mathbb{F}_{e}$. Assume that $\Delta$ is contained in a smooth curve $\Gamma$ of $\mathbb{F}_{e}$. Let $a C_{0}+b f \in \operatorname{Pic}\left(\mathbb{F}_{e}\right)$. If $h^{1}\left(\mathbb{F}_{e}, a C_{0}+b f-\Gamma\right)=0$ then $h^{0}\left(\mathbb{F}_{e}, \mathcal{I}_{\Delta}\left(a C_{0}+b f\right)\right)=h^{0}\left(\mathbb{F}_{e}, a C_{0}+b f-\Gamma\right)+$ $h^{0}\left(\Gamma,\left(a C_{0}+b f\right)_{\mid \Gamma}-\Delta\right)$.

Proof. Let us call $D:=a C_{0}+b f$. In our assumptions we have the following commutative diagram:

$$
\begin{aligned}
& 0 \\
& H^{0}\left(\mathbb{F}_{e}, D-\Gamma\right)
\end{aligned}
$$

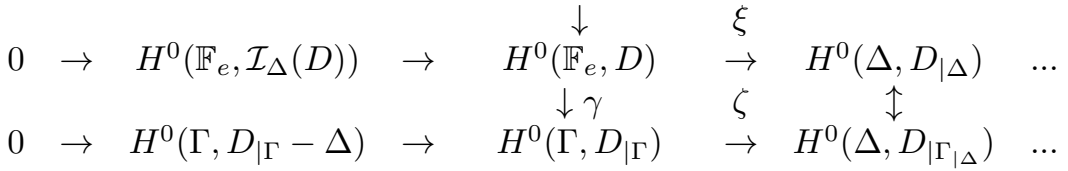

$$
\begin{aligned}
& \downarrow
\end{aligned}
$$

Note that $H^{0}\left(\Delta, D_{\mid \Delta}\right) \simeq H^{0}\left(\Delta, D_{\mid \Gamma_{\mid \Delta}}\right) \simeq \mathbb{C}^{\delta}$, moreover $\gamma(\operatorname{ker} \xi)=\operatorname{ker} \zeta$. Hence: $h^{0}\left(\Gamma, D_{\mid \Gamma}-\Delta\right)=h^{0}\left(\mathbb{F}_{e}, \mathcal{I}_{\Delta}(D)\right)-\operatorname{dim}\left\{H^{0}\left(\mathbb{F}_{e}, \mathcal{I}_{\Delta}(D)\right) \cap H^{0}\left(\mathbb{F}_{e}, D-\Gamma\right)\right\}$ $h^{0}\left(\Gamma, D_{\mid \Gamma}-\Delta\right)=h^{0}\left(\mathbb{F}_{e}, \mathcal{I}_{\Delta}(D)\right)-h^{0}\left(\mathbb{F}_{e}, D-\Gamma\right)$, see Proposition 2 of $[\mathrm{A}-\mathrm{T}]$.

Corollary 1. In Proposition 3 let us assume that $\Gamma \in\left|C_{0}+\beta f\right|$ and $a=1$, then $h^{0}\left(\mathbb{F}_{e}, \mathcal{I}_{\Delta}(D)\right)=\max \{b-\beta+1,0\}+\max \{b+\beta-e-\delta+1,0\}$. In fact, in this case, $D \Gamma=b+\beta-e$ and $h^{0}\left(\mathbb{F}_{e}, D-\Gamma\right)=h^{0}\left(\mathbb{F}_{e},(b-\beta) f\right)=h^{0}\left(\mathbb{P}^{1}, \mathcal{O}_{\mathbb{P}^{1}}(b-\beta)\right)$.

With the help of Proposition 3 and Corollary 1 we can prove the following Theorem giving the values of $\varphi(x)$ when $\Delta$ is contained in a smooth section.

Theorem 1. Let $\Delta$ be a set of $\delta \geq 1$ distinct points on a rational ruled surface $\mathbb{F}_{e}$. Assume that $\Delta$ is contained in a smooth rational curve $\Gamma \in\left|C_{0}+\beta f\right|, \beta \geq e$. Then:

if $0<x<e$ and $x \leq \delta-1, \varphi(x)=0$;

if $0<x<e$ and $x>\delta-1, \varphi(x)=x+1-\delta$;

if $x \geq e, x \geq \beta-1$ and $x \geq e+\delta-\beta, \varphi(x)=2 x+2-e-\delta>0$;

if $x \geq e, x \geq \beta-1$ and $x<e+\delta-\beta, \varphi(x)=x-\beta+1 \geq 0$;

if $e \leq x<\beta-1$ and $x<e+\delta-\beta, \varphi(x)=0$;

if $e \leq x<\beta-1$ and $x \geq e+\delta-\beta, 0 \leq \varphi(x) \leq x+\beta-e-\delta+1$. 
Proof. If $0<x<e$ we know that $\left|C_{0}+x f\right|=C_{0}+|x f|$. Moreover, as the $\delta$ points belong to a smooth section of $\mathbb{F}_{e}$, it follows that every fibre of $\mathbb{F}_{e}$ contains at most one point of $\Delta$. Hence $\varphi(x):=h^{0}\left(\mathbb{F}_{e}, \mathcal{I}_{\Delta}\left(C_{0}+x f\right)\right)=h^{0}\left(\mathbb{P}^{1}, \mathcal{O}_{\mathbb{P}^{1}}(x-\delta)\right)$ and we can conclude.

If $x \geq e$, and $x \geq \beta-1$ we have that $h^{1}\left(\mathbb{F}_{e}, C_{0}+x f-\Gamma\right)=h^{1}\left(\mathbb{F}_{e},(x-\beta) f\right)=0$, hence we can apply Corollary 1 ; as $x-\beta \geq-1$, we get:

$\varphi(x)=x-\beta+1+(-e+x+\beta-\delta+1)=2 x+2-e-\delta>0$ if $x \geq e+\delta-\beta$

$\varphi(x)=x-\beta+1+0=x-\beta+1 \geq 0$ if $x<e+\delta-\beta$.

If $e \leq x<\beta-1$ we have that $h^{0}\left(\mathbb{F}_{e}, C_{0}+x f-\Gamma\right)=h^{0}\left(\mathbb{F}_{e},(x-\beta) f\right)=0$ and we can consider a diagram as in Proposition 3 where $D:=C_{0}+x f$ :

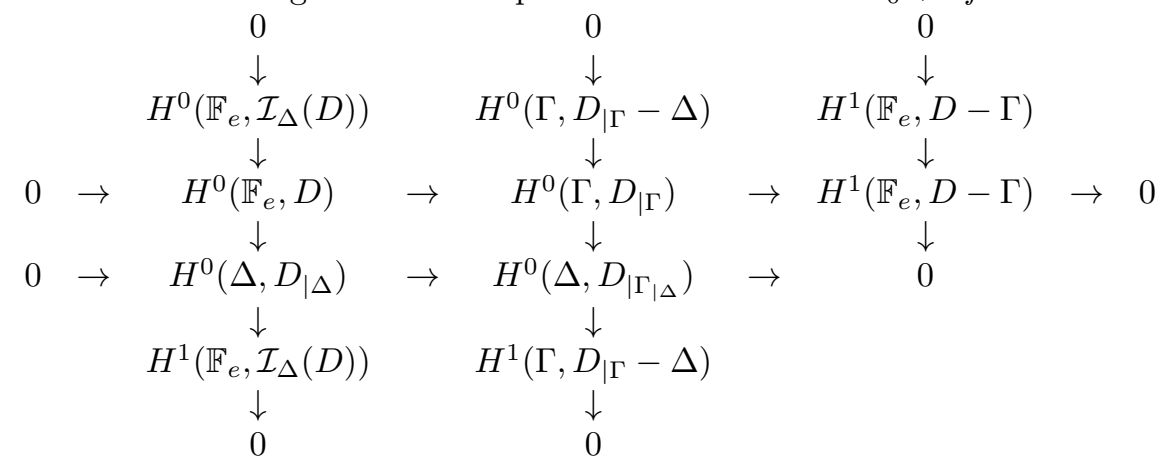

By the snake lemma we get that, if $x<e+\delta-\beta$ then $h^{0}\left(\Gamma, D_{\mid \Gamma}-\Delta\right)=0$, hence $\varphi(x)=0$. If $x \geq e+\delta-\beta$ we have that $0 \leq \varphi(x) \leq h^{0}\left(\Gamma, D_{\mid \Gamma}-\Delta\right)=x+\beta-e-\delta+1$, but no more precise results can be gotten by the diagram.

Theorem 1 allows to describe the function $\varphi(x)$ in some detail, however, when $x \geq e$, its graph depends on the relative position of the involved integers. To get a more precise description of $\varphi(x)$, useful to prove (or disprove) the general position of $\Delta$, it is better to distinguish four cases when $x \geq e$.

Case 1) : $\beta=e$;

$1(i): \delta \leq e$; then $\varphi(x)=2 x+2-e-\delta$ for $x \geq e$;

$1($ ii $): \delta>e$; then $\varphi(x)=x-e+1$ for $x \in[e, \delta-1]$ and $\varphi(x)=2 x+2-e-\delta$ for $x \geq \delta$.

Case 2) : $\beta=e+1$;

$2(i): \delta \leq e+1$; then $\varphi(x)=2 x+2-e-\delta$ for $x \geq e$;

$2(i i): \delta>e+1$; then $\varphi(x)=x-e$ for $x \in[e, \delta-2]$ and $\varphi(x)=2 x+2-e-\delta$ for $x \geq \delta-1$.

Case 3$): \beta=e+2$;

$3(i): \delta \leq e+3$; then $0 \leq \varphi(e) \leq e+3-\delta$ and $\varphi(x)=2 x+2-e-\delta$ for $x \geq e+1$;

$3(i i): \delta>e+3$; then $\varphi(e)=0, \varphi(x)=x-e-1$ for $x \in[e+1, \delta-3]$ and $\varphi(x)=2 x+2-e-\delta$ for $x \geq \delta-2$.

Case 4) : $\beta \geq e+3$;

$4(i): e+\delta-\beta \leq e$ i.e. $\delta \leq \beta$; then $0 \leq \varphi(x) \leq x+\beta-e-\delta+1$ for $x \in[e, \beta-2]$ and $\varphi(x)=2 x+2-e-\delta$ for $x \geq \beta-1$;

4(ii) : e $<e+\delta-\beta<\beta-1$, i.e. $\delta>\beta$ and $e+\delta<2 \beta-1$; then $\varphi(x)=0$ for $x \in[e, e+\delta-\beta-1]$ and $0 \leq \varphi(x) \leq x+\beta-e-\delta+1$ for $x \in[e+\delta-\beta, \beta-2]$ and $\varphi(x)=2 x+2-e-\delta$ for $x \geq \beta-1$;

4(iii) : $e<e+\delta-\beta \leq \beta-1$, i.e. $\delta>\beta$ and $e+\delta=2 \beta-1$; then $\varphi(x)=0$ for $x \in[e, \beta-2]$ and $\varphi(x)=2 x+2-e-\delta$ for $x \geq \beta-1$; 
$4(i v): e+\delta-\beta>\beta-1$, i.e. $e+\delta>2 \beta-1$; then $\varphi(x)=0$ for $x \in[e, \beta-2]$ and $\varphi(x)=x-e-1$ for $x \in[\beta-1, e+\delta-\beta-1]$ and $\varphi(x)=2 x+2-e-\delta$ for $x \geq e+\delta-\beta$.

Now we can prove the following Corollary that, in the sequel, will be used to check the general position of sets of points $\Delta$ when $\Delta \cap C_{0}=\emptyset$.

Corollary 2. Let $\Delta$ be a set of $\delta \geq 1$ distinct points on a rational ruled surface $\mathbb{F}_{e}$ contained in a smooth rational curve $\Gamma \in\left|C_{0}+\beta f\right|, \beta \geq e$. Let us assume that $\Delta \cap C_{0}=\emptyset$. Then $\Delta$ is in general position, according to Definition 1 , if and only if:

a) $\beta=e, 1 \leq \delta \leq e$.

b) $\beta=e+1,1 \leq \delta \leq e+3$.

c) $\beta=e+2,1 \leq \delta \leq e+2$ and $\varphi(e)=e+2-\delta$.

d) $\beta=e+2, e+3 \leq \delta \leq e+5$.

e) $\beta \geq e+3, e+2 \leq \delta \leq \beta$ and $\varphi(x)$ is the expected value for every $x \in[e, \beta-2]$.

f) $\beta \geq e+3, \delta>\beta, e+\delta<2 \beta-1$ and $\varphi(x)$ is the expected value for $x \in$ $[e+\delta-\beta, \beta-2]$.

g) $\beta \geq e+3, \delta>\beta, 2 \beta-1 \leq e+\delta \leq 2 \beta+1$.

Proof. The assumption $\Delta \cap C_{0}=\emptyset$ implies that $\varphi(0)=0$, i.e. the expected value for points in general position. When $0<x<e$, Theorem 1 says that $\Delta$ is a set of points in general position. So we have only to consider the cases when $x \geq e$. To this aim, we remark that $\Delta \subset \Gamma$, hence $\varphi(\beta)>0$. It follows that a necessary condition is $2 \beta+2-e-\delta>0$, i.e. $e+\delta-\beta \leq \beta+1$.

Now we can use Theorem 1 and the following description of $\varphi(x)$ given by the above cases 1$), \ldots, 4)$, to decide, case by case, the necessary and sufficient conditions for the general position of $\Delta$ when $x \geq e$ and $e+\delta-\beta \leq \beta+1$. Let us recall that $\Delta$ is in general position according to Definition 1 if and only if $\varphi(x)=2 x+2-e-\delta$ if $x>\frac{e+\delta}{2}-1$ and $\varphi(x)=0$ if $x \leq \frac{e+\delta}{2}-1$. Let us write these conditions in a more detailed way:

if $\delta \leq e+2, \varphi(x)=2 x+2-e-\delta$ for $x \geq e$;

if $\delta>e+2$ and $e+\delta$ is odd, $\varphi(x)=0$ for $x \in\left[e, \frac{e+\delta-3}{2}\right]$ and $\varphi(x)=2 x+2-e-\delta$ for $x \geq \frac{e+\delta-1}{2}$;

if $\delta>e+2$ and $e+\delta$ is even, $\varphi(x)=0$ for $x \in\left[e, \frac{e+\delta-2}{2}\right]$ and $\varphi(x)=2 x+2-e-\delta$ for $x \geq \frac{e+\delta}{2}$.

Let us examine cases 1$), \ldots, 4)$.

Case 1$)$. In the subcase $1(i), \Delta$ is in general position and we get $a$ ). In the subcase $1(i i), \Delta$ is not in general position; for instance: $\varphi(e)$ is not the expected value 0 when $\delta \geq e+2$ and $\varphi(e+1)$ is not the expected value 1 when $\delta=e+1$.

Case 2$)$. In the subcase $2(i), \Delta$ is in general position; in the subcase $2(i i), \Delta$ is in general position when $\delta=e+2, e+3$ and we get $b)$. On the contrary, $\Delta$ is not in general position when $\delta \geq e+4$, for instance $\varphi(e+1)$ is not the expected value 0 .

Case 3$)$. In the subcase $3(i), \varphi(x)$ is always the expected value with the possible exception of $x=e$, hence $\Delta$ is in general position if and only if $\varphi(e)$ is the expected value, i.e. $e+2-\delta$ if $\delta \leq e+2$ and 0 if $\delta=e+3$. When $\delta \leq e+2$ it must be $\varphi(e)=e+2-\delta$ and we get $c$ ); when $\delta=e+3$ we have that $\varphi(e)=0$ and we get $d)$. In the subcase $3(i i)$, if $\delta=e+4$ or $\delta=e+5, \varphi(x)$ is always the expected value and we get $d$ ). There are no other possibilities because the necessary condition $e+\delta-\beta \leq \beta+1$ in this case becomes $\delta \leq e+5$. 
Case 4$)$. In the subcase $4(i), \varphi(x)$ is the expected value for $x \geq \beta-1$, so we have only to check for $x \in[0, \beta-2]$ and we get $e)$. In the subcase $4(i i), \varphi(x)$ is the expected value for $x \in[e, e+\delta-\beta-1]$ and for $x \geq \beta-1$, so we have only to check for $x \in[e+\delta-\beta, \beta-2]$ and we get $f)$. In the subcase $4(i i i), \varphi(x)$ is always the expected value and we get $g)$. In the subcase $4(i v)$ the necessary condition implies that $e+\delta-\beta=\beta$ or $e+\delta-\beta=\beta+1$, in both cases $\varphi(x)$ is the expected value and we get $g$ ).

Theorem 1 does not succeed in calculating $\varphi(x)$ when $x \geq e$ and $x \in[e+\delta-$ $\beta, \beta-2]$; note that this situation includes the three cases $c$ ), $e$ ) and $f$ ) of Corollary 2 where the general position of $\Delta$ depends on the value of $\varphi(x)$. To get an answer in any case it would be sufficient to calculate $\varphi(x)$ when $\beta \geq e+2$ and $x \in[e, \beta-2]$. In this range there is another way to calculate $\varphi(x)$ and we can be more precise in some cases. This is the motivation for the next Proposition 4 (not used in the sequel) in which we will summarize some information. Let us start with the following

Lemma 1. Let $\mathbb{F}_{e}$ be a rational ruled surface embedded in $\mathbb{P}^{N}$ by the very ample line bundle $C_{0}+\beta f$ with $\beta>e>0$ and $N=h^{0}\left(\mathbb{F}_{e}, C_{0}+\beta f\right)=2 \beta+1-e$. Let $\eta$ be a positive integer. Let us consider any set of $\eta$ fibres of $\mathbb{F}_{e}$ embedded as a scroll in some $\mathbb{P}^{N}$, hence the $\eta$ fibres are embedded as lines. Let $Y_{\eta}$ be the linear space spanned by these lines in $\mathbb{P}^{N}$. Then:

if $0 \leq \eta \leq \beta-e, \operatorname{dim}\left(Y_{\eta}\right)=2 \eta-1$, (we put $\operatorname{dim}(\emptyset):=-1$ )

if $\beta-e<\eta \leq \beta, \operatorname{dim}\left(Y_{\eta}\right)=\beta-e+\eta$,

if $\beta<\eta, \operatorname{dim}\left(Y_{\eta}\right)=N=2 \beta+1-e$.

Proof. Obviously $h^{0}\left(\mathbb{F}_{e}, C_{0}+\beta f-\eta f\right)=0$ if $\operatorname{dim}\left(Y_{\eta}\right)=N$, while $h^{0}\left(\mathbb{F}_{e}, C_{0}+\beta f-\right.$ $\eta f)=N-1-\operatorname{dim}\left(Y_{\eta}\right)+1=N-\operatorname{dim}\left(Y_{\eta}\right)$ because $h^{0}\left(\mathbb{F}_{e}, C_{0}+\beta f-\eta f\right)$ is the vectorial dimension of the projective space dual of $Y_{\eta}$. Now $h^{0}\left(\mathbb{F}_{e}, C_{0}+\beta f-\eta f\right)=$ $h^{0}\left(\mathbb{P}^{1}, \mathcal{O}_{\mathbb{P}^{1}}(-e+\beta-\eta)\right)+h^{0}\left(\mathbb{P}^{1}, \mathcal{O}_{\mathbb{P}^{1}}(\beta-\eta)\right)$.

If $1 \leq \eta \leq \beta-e$ we have $2 \beta+1-e-\operatorname{dim}\left(Y_{\eta}\right)=-e+\beta-\eta+1+\beta-\eta+1$, hence $\operatorname{dim}\left(Y_{\eta}\right)=2 \eta-1$. If $\beta-e<\eta \leq \beta$ we have $2 \beta+1-e-\operatorname{dim}\left(Y_{\eta}\right)=\beta-\eta+1$, hence $\operatorname{dim}\left(Y_{\eta}\right)=\beta-e+\eta$. If $\beta<\eta$ we have $h^{0}\left(\mathbb{F}_{e}, C_{0}+\beta f-\eta f\right)=0$, hence $\operatorname{dim}\left(Y_{\eta}\right)=N$.

Proposition 4. Let $\mathbb{F}_{e}$ be a rational ruled surface embedded as a scroll in $\mathbb{P}^{N}$ by the very ample line bundle $C_{0}+\beta f$ with $\beta>e>0$, where $N=h^{0}\left(\mathbb{F}_{e}, C_{0}+\beta f\right)-1=$ $2 \beta+1-e$. Let $\Delta$ be a set of $\delta \geq 1$ distinct points belonging to a smooth curve $\Gamma \in\left|C_{0}+\beta f\right|$ and let $Y_{\delta}$ be the linear space spanned by these points in $\mathbb{P}^{N}$. Let $Y_{\eta}$ be the linear space spanned in $\mathbb{P}^{N}$ by $\eta$ generic lines of the scroll. Let $\lambda:=\operatorname{dim}\left(Y_{\delta} \cap Y_{\eta}\right)$. Then:

i) $\varphi(\beta)=2 \beta+2-e-\delta$ if $2 \beta+2>e+\delta$;

$\varphi(\beta)=1$ if $2 \beta+2 \leq e+\delta$.

ii) $\Delta$ can be in general position only if $e+\delta<2 \beta+2$ i.e. $e+\delta \leq 2 \beta+1$.

iii) When $e+\delta \leq 2 \beta+1$, for any $e \leq x<\beta$ we have:

$\varphi(x)=2 x+2-e-\delta+(\lambda+1)$ if $2 x \geq \delta+e-3-\lambda$;

$\varphi(x)=0$ if $2 x \leq \delta+e-3-\lambda$.

iv) When $e+\delta \leq 2 \beta+1$, for any $e \leq x<\beta$ we have that $\varphi(x)$ is the expected value if $\lambda=-1$.

v) When $e+\delta \leq 2 \beta+1$, for any $e \leq x<\beta$ we have that, if $\frac{\delta+e-3}{2} \in[e, \beta-2]$, then $\varphi(x)=0$ for any $x \in\left[e, \frac{\delta+e-3}{2}\right]$ and this is the expected value for $\varphi(x)$. 
Proof. Let us start with general remarks. $\Gamma$ is a generic hyperplane section of the surface, hence it is a non degenerate smooth rational curve of degree $N-1=2 \beta-e$ in a projective space of the same dimension. Hence:

$\operatorname{dim}\left(Y_{\delta}\right)=\delta-1$ if $1 \leq \delta \leq N=2 \beta-e+1$ and $\operatorname{dim}\left(Y_{\delta}\right)=N-1$ if $\delta>N$.

Let us put $x=\beta-\eta$ with $0 \leq \eta \leq \beta-e$, so that $\varphi(x)=h^{0}\left(\mathbb{F}_{e}, \mathcal{I}_{\Delta}\left(C_{0}+\beta f-\eta f\right)\right)$ is the vectorial dimension of the projective space dual of $\left\langle Y_{\delta}, Y_{\eta}\right\rangle$. Hence: $\varphi(x)=$ $N-1-\operatorname{dim}\left\langle Y_{\delta}, Y_{\eta}\right\rangle+1=N-\operatorname{dim}\left(Y_{\delta}\right)-\operatorname{dim}\left(Y_{\eta}\right)+\lambda$ if $\operatorname{dim}\left(Y_{\delta}\right)+\operatorname{dim}\left(Y_{\eta}\right)-\lambda \leq N$; $\varphi(x)=0$ if $\operatorname{dim}\left(Y_{\delta}\right)+\operatorname{dim}\left(Y_{\eta}\right)-\lambda \geq N$. Let us call $(*)$ these formulas.

Now we can prove the five items.

i) From formulas $(*)$ we get: $\varphi(\beta)=N-\operatorname{dim}\left(Y_{\delta}\right)$ because in this case $\eta=0$, $\operatorname{dim}\left(Y_{\eta}\right)=\lambda=-1$ and $\operatorname{dim}\left(Y_{\delta}\right) \leq N-1$ in any case. Hence $\varphi(\beta)=N-\delta+1=$ $2 \beta+2-e-\delta$ if $\delta \leq 2 \beta-e+1$, i.e. if $2 \beta+2>e+\delta$ and $\varphi(\beta)=1$ if $\delta>2 \beta-e+1$, i.e. if $2 \beta+2 \leq e+\delta$.

ii) If $\Delta$ is in general position $\varphi(\beta)=0$ if $2 \beta+2 \leq e+\delta$, hence $\Delta$ can be in general position only if $2 \beta+2>e+\delta$, i.e. if $e+\delta \leq 2 \beta+1$.

iii) In this case $\operatorname{dim}\left(Y_{\eta}\right)=2 \eta-1$ by Lemma 1 because $0<\eta \leq \beta-e$ and $\operatorname{dim}\left(Y_{\delta}\right)=\delta-1$ because $e+\delta \leq 2 \beta+1$. From formulas $(*)$ we get $\varphi(x)=2 \beta-e+$ $1-(\delta-1)-(2 \eta-1)+\lambda=2 x+2-e-\delta+(\lambda+1)$ if $(\delta-1)+(2 \eta-1)-\lambda \leq N$ i.e. $\delta-1+2 \beta-2 x-1-\lambda \leq 2 \beta-e+1$, i.e. $2 x \geq \delta+e-3-\lambda$. We get $\varphi(x)=0$ if $(\delta-1)+(2 \eta-1)-\lambda \geq N$ i.e. $2 x \leq \delta+e-3-\lambda$.

iv) If $\lambda=-1$ the value of $\varphi(x)$ calculated in $i i i)$ is the expected one.

$v$ ) When $\operatorname{dim}\left(Y_{\delta}\right)+\operatorname{dim}\left(Y_{\eta}\right) \geq N$ then $\lambda=\operatorname{dim}\left(Y_{\delta}\right)+\operatorname{dim}\left(Y_{\eta}\right)-N$. In our range $\operatorname{dim}\left(Y_{\delta}\right)=\delta-1$ and $\operatorname{dim}\left(Y_{\eta}\right)=2 \eta-1$ as in $\left.i i\right)$. Hence, when $\delta-1+2 \eta-1 \geq N$, i.e. when $\delta+e-3 \geq 2 x$, then $\lambda=\delta+e-3-2 x$. By using $i i i)$ we get $\varphi(x)=0$, therefore if $\frac{\delta+e-3}{2} \in[e, \beta-2]$ we get $\varphi(x)=0$ for any $x \in\left[e, \frac{\delta+e-3}{2}\right]$ and this is the expected value for $\varphi(x)$ by looking at Remark 1 .

Remark 2. Let us remark that the necessary upper bound given by Proposition 4 ii) is satisfied by all the possibilities listed in Corollary 2.

By the previous results one could suspect that a set $\Delta$ of $\delta$ distinct points belonging to a smooth section $\Gamma \in\left|C_{0}+\beta f\right|$ with $\beta>e$ could be always in general position provided that $\Delta \cap C_{0}=\emptyset$. This is not the case. We will give a pair of examples in which we will assume $\Delta \cap C_{0}=\emptyset$.

Example 1. Let us choose: $e=1, \beta=\delta=3$. By Corollary $2 c), \varphi(1)=0$ is the only value to check to prove the generic position of a set $\Delta$ of 3 distinct points belonging to a curve $\Gamma \in\left|C_{0}+3 f\right|$. In this case, the very ample linear system $\left|C_{0}+3 f\right|$ embeds the surface $\mathbb{F}_{1}$ as a scroll in $\mathbb{P}^{6}$ and the scroll can be constructed as follows: take a disjoint pair of projective spaces of dimension 2 and 3 in $\mathbb{P}^{6}$; take rational normal curves $C_{2}$ of degree 2 and $C_{3}$ of degree 3 , respectively, in such spaces; fix a regular map between $C_{2}$ and $C_{3}$ : the ruled surface is given by the lines joining the corresponding points of $C_{2}$ and $C_{3}$.

By choosing suitable coordinates in $\mathbb{P}^{6}$, to choose the above regular map is equivalent to parametrize the two rational curves with the same copy of $\mathbb{P}^{1}$. In this projective copy let us choose affine coordinates $(\tau: 1)$ in such a way that the points of $C_{2}$ have coordinates $\left(\tau^{2}: \tau: 1: 0: 0: 0: 0\right)$ and the corresponding points of $C_{3}$ have coordinates $\left(0: 0: 0: \tau^{3}: \tau^{2}: \tau: 1\right)$. Now we can choose five distinct values for $\tau: a, b, c, t, s$ in such a way that the three points of $\Delta$ have coordinates:

$$
h\left(a^{2}: a: 1: 0: 0: 0: 0\right)+\left(0: 0: 0: a^{3}: a^{2}: a: 1\right)=\left(h a^{2}: h a: h: a^{3}: a^{2}: a: 1\right)
$$




$$
\begin{aligned}
& k\left(b^{2}: b: 1: 0: 0: 0: 0\right)+\left(0: 0: 0: b^{3}: b^{2}: b: 1\right)=\left(k b^{2}: k b: k: b^{3}: b^{2}: b: 1\right) \\
& l\left(c^{2}: c: 1: 0: 0: 0: 0\right)+\left(0: 0: 0: c^{3}: c^{2}: c: 1\right)=\left(l c^{2}: l c: l: c^{3}: c^{2}: c: 1\right)
\end{aligned}
$$

by using affine coordinates $h, k, l$, respectively, on the three lines of the scroll passing through $\Delta$. The other two values $t, s$ of $\tau$ determine two lines of the scroll as follows:

$\left\langle\left(t^{2}: t: 1: 0: 0: 0: 0\right),\left(0: 0: 0: t^{3}: t^{2}: t: 1\right)\right\rangle$ and

$\left\langle\left(s^{2}: s: 1: 0: 0: 0: 0\right),\left(0: 0: 0: s^{3}: s^{2}: s: 1\right)\right\rangle$.

Note that the three points of $\Delta$ are surely independent in $\mathbb{P}^{6}$ for any choice of $h, k, l$, hence $\Delta$ belongs to (at least one) hyperplane section $\Gamma$.

In this way $\varphi(1)=h^{0}\left(\mathbb{F}_{1}, \mathcal{I}_{\Delta}\left(C_{0}+f\right)\right)$ is the vectorial dimension of the projective space of hyperplanes of $\mathbb{P}^{6}$ passing through $\Delta$ and the two above lines. Hence $\varphi(1)=0$ if and only if the following seven points of $\mathbb{P}^{6}$ are independent:

$\left(h a^{2}: h a: h: a^{3}: a^{2}: a: 1\right)$

$\left(k b^{2}: k b: k: b^{3}: b^{2}: b: 1\right)$

$\left(l c^{2}: l c: l: c^{3}: c^{2}: c: 1\right)$

$\left(t^{2}: t: 1: 0: 0: 0: 0\right)$

$\left(0: 0: 0: t^{3}: t^{2}: t: 1\right)$

$\left(s^{2}: s: 1: 0: 0: 0: 0\right)$

$\left(0: 0: 0: s^{3}: s^{2}: s: 1\right)$.

If we consider the $(7,7)$ matrix given by the coordinates of the seven points we have that its determinant is

$-(s-c)(s-b)(s-a)(t-c)(t-b)(t-a)(t-s)^{2}[(h-k) c+(k-b) a+(l-h) b]$.

As the five values $a, b, c, t, s$ are distinct, we have that $\varphi(1)=0$ if and only if $(h-k) c+(k-l) a+(l-h) b \neq 0$. The conclusion is that, for a generic choice of $\Delta$ the three points are in general position on $\mathbb{F}_{1}$, however there exists a divisor of degree $(1,1,1)$ in $\left(\mathbb{P}^{1}\right)^{\times 3}$ such that $\Delta$ is not in general position if and only if $\Delta$ belongs to this divisor. Note that this conclusion is independent of the choice of the five distinct values $a, b, c, t, s$.

Example 2. Let us choose: $e=2, \beta=5, \delta=6$. By Corollary $2 f), \varphi(3)=0$ is the only value to check to prove the generic position of a set $\Delta$ of 6 distinct points belonging to a curve $\Gamma \in\left|C_{0}+5 f\right|$. In this case the very ample linear system $\left|C_{0}+5 f\right|$ embeds $\mathbb{F}_{2}$ as a scroll in $\mathbb{P}^{9}$ and the scroll can be constructed as follows: take a disjoint pair of projective spaces of dimension 3 and 5 in $\mathbb{P}^{9}$; take rational normal curves $C_{3}$ of degree 3 and $C_{5}$ of degree 5 , respectively, in such spaces; fix a regular map between $C_{3}$ and $C_{5}$ : the ruled surface is given by the lines joining the corresponding points of $C_{3}$ and $C_{5}$.

By choosing suitable coordinates in $\mathbb{P}^{9}$, to choose the above regular map is equivalent to parametrize the two rational curves with the same copy of $\mathbb{P}^{1}$. In this projective copy let us choose affine coordinates $(\tau: 1)$ in such a way that the points of $C_{3}$ have coordinates $\left(\tau^{3}: \tau^{2}: \tau: 1: 0: 0: 0: 0: 0: 0\right)$ and the corresponding points of $C_{5}$ have coordinates $\left(0: 0: 0: 0: \tau^{5}: \tau^{4}: \tau^{3}: \tau^{2}: \tau: 1\right)$. Now we can choose eight distinct values for $\tau: a_{1}, \ldots, a_{6}, t, s$ in such a way that the six points of $\Delta$ have coordinates as in Example 1 (we use affine coordinates $h_{i}$ on every line, $i=1, \ldots, 6)$ :

$\left(h_{i} a_{i}^{3}: h_{i} a_{i}^{2}: h_{i} a_{i}: h_{i}: a_{i}^{5}: a_{i}^{4}: a_{i}^{3}: a_{i}^{2}: a_{i}: 1\right)$

and the other two values $t, s$ determine two lines as follows:

$\left\langle\left(t^{3}: t^{2}: t: 1: 0: 0: 0: 0: 0: 0\right),\left(0: 0: 0: 0: t^{5}: t^{4}: t^{3}: t^{2}: t: 1\right)\right\rangle$ and

$$
\left\langle\left(s^{3}: s^{2}: s: 1: 0: 0: 0: 0: 0: 0\right),\left(0: 0: 0: 0: s^{5}: s^{4}: s^{3}: s^{2}: s: 1\right)\right\rangle .
$$


Note that the six points of $\Delta$ are surely independent in $\mathbb{P}^{9}$ for any choice of $h_{i}$, hence $\Delta$ belongs to (at least one) hyperplane section $\Gamma$.

In this way $\varphi(3)=h^{0}\left(\mathbb{F}_{2}, \mathcal{I}_{\Delta}\left(C_{0}+3 f\right)\right)$ is the vectorial dimension of the projective space of hyperplanes of $\mathbb{P}^{9}$ passing through $\Delta$ and the two above lines. Hence $\varphi(3)=0$ if and only if the following ten points of $\mathbb{P}^{9}$ are independent:

$\left(h_{i} a_{i}^{3}: h_{i} a_{i}^{2}: h_{i} a_{i}: h_{i}: a_{i}^{5}: a_{i}^{4}: a_{i}^{3}: a_{i}^{2}: a_{i}: 1\right), i=1, \ldots, 6$

$\left(t^{3}: t^{2}: t: 1: 0: 0: 0: 0: 0: 0\right)$

$\left(0: 0: 0: 0: t^{5}: t^{4}: t^{3}: t^{2}: t: 1\right)$

$\left(s^{3}: s^{2}: s: 1: 0: 0: 0: 0: 0: 0\right)$

$\left(0: 0: 0: 0: s^{5}: s^{4}: s^{3}: s^{2}: s: 1\right)$.

If we calculate the determinant of the $(10,10)$ matrix given by the coordinates of the ten points, by a computer algebra system, we get that the determinant is the product of many non zero factors, due to the distinct choice of the eight values, and a polynomial $p\left(h_{i}, a_{i}\right)$ of degree two with respect to the variables $h_{i}$. So that we have that $\varphi(3)=0$ if and only if $p\left(h_{i}, a_{i}\right) \neq 0$. The conclusion is that, for a generic choice of $\Delta$ the five points are in general position on $\mathbb{F}_{2}$, however there exists a divisor of degree $(2,2,2,2,2)$ in $\left(\mathbb{P}^{1}\right)^{\times 5}$ such that $\Delta$ is not in general position if and only if $\Delta$ belongs to this divisor. Note that this conclusion is independent of the choice of the eight distinct values $\left\{a_{i}, t, s\right\}$.

\section{Very AMPleness AND GENERAL POSITION, FIRST EXAMPLES}

As we have promised in $\S 2$ let us consider rank 2 vector bundles $E$ over $\mathbb{F}_{e}$ sitting in an exact sequence as $(\epsilon)$ with $L \sim C_{0}+\alpha f, M \sim C_{0}+\beta f$ and $Z=\emptyset$. We can assume that $E \neq C_{0}+\alpha f \oplus C_{0}+\beta f$, otherwise the very ampleness of $E$ is simply equivalent to the very ampleness of both $C_{0}+\alpha f$ and $C_{0}+\beta f$; hence we can assume that $\operatorname{Ext}^{1}(M, L) \neq 0$, i.e. that $H^{1}\left(\mathbb{F}_{e},(\alpha-\beta) f\right) \neq 0$, i.e. that $\alpha \leq \beta-2$. Moreover we assume that $\alpha \geq e$ in such a way that the generic element of $|L|$ is a smooth curve and $L M$ is in fact the number of points of intersection of smooth rational curves on the surface. Note that $\alpha \geq e$ implies $H^{1}\left(\mathbb{F}_{e}, L\right)=0$, hence $H^{0}\left(\mathbb{F}_{e}, E\right) \rightarrow H^{0}\left(\mathbb{F}_{e}, M\right)$ is surjective and moreover, for any generic section $s$ of $E, \Gamma_{s}$ is a rational smooth curve by Proposition 2 iii).

In the above assumptions for $E$, the following Proposition lists all the cases in which, if $E$ is very ample, the zero locus of a generic section $s$ of $E$ can be in general position when $\alpha \geq e$.

Proposition 5. Let $E$ be a very ample rank 2 vector bundle over $\mathbb{F}_{e}$ sitting in an exact sequence as follows:

$$
0 \rightarrow C_{0}+\alpha f \rightarrow E \rightarrow C_{0}+\beta f \rightarrow 0
$$

and let $\Delta:=(s)_{0}$ be the zero locus of a generic section $s$ of $E$. Assume that $\delta:=$ length $(\Delta) \geq 1$ and that $0<e \leq \alpha \leq \beta-2$. Then $\Delta$ can be in general position only if:

i) $\alpha=e, \delta=\beta=e+2$;

and in this case $\Delta$ is in fact in general position if and only if $\Delta \cap C_{0}=\emptyset$ and $\varphi(\alpha)=0$.

ii) $\alpha=e, \delta=\beta \geq e+3$

and in this case $\Delta$ is in fact in general position if and only if $\Delta \cap C_{0}=\emptyset$ and $\varphi(x)$ is the expected value for every $x \in[\alpha, \beta-2]$.

iii) $e<\alpha \leq \beta-2, \delta>\beta \geq e+3$; 
and in this case $\Delta$ is in fact in general position if and only if $\Delta \cap C_{0}=\emptyset$ and $\varphi(x)$ is the expected value for every $x \in[\alpha, \beta-2]$.

Proof. As $E$ is very ample then $C_{0}+\beta f$ must be ample, hence $\beta>e$, moreover $\Delta$ is given by a set of $\delta=-e+\alpha+\beta$ distinct points, belonging to a smooth rational curve $\Gamma_{s}$ by Proposition $2 \mathrm{ii}$ ) and $\mathrm{iii}$ ), hence we can use Corollary 2 to decide whether $\Delta$ can be in general position, always assuming that $\Delta \cap C_{0}=\emptyset$.

Of course cases $a$ ) and $b$ ) do not occur. In case $c$ ), $\Delta$ can be in general position when $\alpha=e$ and $\delta=\beta=e+2$; in fact $\Delta$ is in general position if and only if $\varphi(\alpha)=0$. Case $d$ ) does not occur. In case $e$ ), $\Delta$ can be in general position when $\alpha=e$ again and $\delta=\beta \geq e+3$; in fact $\Delta$ is in general position if and only if $\varphi(x)$ is the expected value for every $x \in[\alpha, \beta-2]$. In case $f), \Delta$ can be in general position when $e<\alpha \leq \beta-2$ and $\delta>\beta \geq e+3$; in fact $\Delta$ is in general position if and only if $\varphi(x)$ is the expected value for every $x \in[\alpha, \beta-2]$. Case $g$ ) does not occur.

Proposition 5 shows that there exist few cases in which, if a rank 2 vector bundle $E$ as above is very ample, then the zero locus of its generic sections can be in general position. The following Theorem proves that $E$ is always very ample in almost all these cases.

Theorem 2. Let $E$ be a rank 2 vector bundle over $\mathbb{F}_{e}$ sitting in an exact sequence as follows:

$$
0 \rightarrow C_{0}+\alpha f \rightarrow E \rightarrow C_{0}+\beta f \rightarrow 0
$$

with $0<e \leq \alpha \leq \beta-2$ and $\alpha+\beta \geq 2 e+4$. Then $E$ is very ample.

Proof. Let us apply Theorem 4.3 of [A-B] with $x=e+2$ and $z=2$ and $W=\emptyset$. Condition $(i)$ is satisfied: $L\left(C_{0}+(e+2) f\right)=\alpha+2>0 ; M\left(C_{0}+(e+2) f\right)=\beta+2>2$. Conditions $(i i)$ and $(v)$ are satisfied because $W=\emptyset$. Conditions (iii) and (iv) are satisfied because $z=2$. Condition (vi) becomes: $\alpha+\beta \geq 2 e+4$.

The only cases listed by Proposition 5 that are not covered by Theorem 2 are the following ones:

$\alpha=e, \beta=\delta=e+2\left(\Delta\right.$ is in general position if and only if $\Delta \cap C_{0}=\emptyset$ and $\varphi(e)=0)$;

$\alpha=e, \beta=\delta=e+3$ ( $\Delta$ is in general position if and only if $\Delta \cap C_{0}=\emptyset$ and $\varphi(e)=0, \varphi(e+1)=1)$.

In this section we want to discuss the very ampleness of $E$ in these cases, by using the general position of $\Delta$, if possible. We will see that the assumption on the generic position of $\Delta$ is useful only taking into account the condition $\Delta \cap C_{0}=\emptyset$.

Firstly, let us state the following Lemmas.

Lemma 2. Let $E$ be a rank 2 vector bundle over $\mathbb{F}_{e}$, let $s$ be any section of $E$ such that the corresponding surface $S \in|T|$ is smooth, then $(s)_{0}$ is a set of distinct points. Moreover, let $\mathcal{E} \simeq \mathcal{O}_{\mathbb{P}^{1}}(h) \oplus \mathcal{O}_{\mathbb{P}^{1}}(k)$ be the restriction of $E$ to $C_{0}$ with $h \leq k$ and $k \geq 0$, then $\sharp\left((s)_{0} \cap C_{0}\right) \leq k$.

Proof. $S$ is the blow up of $\mathbb{F}_{e}$ at $\sharp\left((s)_{0}\right)$ distinct points (see [B-S] Theorem 11.1.2). Let $Y$ be the ruled surface $\mathbb{P}(\mathcal{E})$ inside $X$; the natural projection $Y \rightarrow C_{0}$ is the restriction of $\pi$. Let $T_{\mid Y} \sim \bar{C}_{0}+k \bar{f}$ be the tautological line bundle of $Y$, where $\bar{C}_{0}$ is the tautogical line bundle of $\mathbb{P}\left(\mathcal{O}_{\mathbb{P}^{1}}(h-k) \oplus \mathcal{O}_{\mathbb{P}^{1}}\right)$ and $\bar{f}$ is the numerical class of a fibre of $Y$. As $S$ is the blow up of $\mathbb{F}_{e}$ at $\sharp\left((s)_{0}\right)$ distinct points, $S \cap Y$ contains 
exactly $\sharp\left((s)_{0} \cap C_{0}\right)$ fibres of $\pi$. But $S \cap Y$ is a curve of $\left|\bar{C}_{0}+k \bar{f}\right|$ and such curves can contain at most $k$ fibres of $\pi$, hence $\sharp\left((s)_{0} \cap C_{0}\right) \leq k$.

Lemma 3. Let $E$ be a rank 2 vector bundle over $\mathbb{F}_{e}$ sitting in an exact sequence as $(\epsilon)$ with $Z=\emptyset$. Let $\mathcal{C}$ be any smooth rational curve of $\mathbb{F}_{e}$ and let us consider the restriction of $(\epsilon)$ to $\mathcal{C}$. There exists a natural map $\rho: \operatorname{Ext}_{\mathbb{F}_{e}}^{1}(M, L) \rightarrow \operatorname{Ext}_{\mathcal{C}}^{1}\left(M_{\mid \mathcal{C}}, L_{\mid \mathcal{C}}\right)$. Unless $\rho$ is the zero map, the generic element of $\operatorname{Ext}_{\mathbb{F}_{e}}^{1}(M, L)$ gives rise to a non zero element of $\operatorname{Ext}_{\mathcal{C}}^{1}\left(M_{\mid \mathcal{C}}, L_{\mid \mathcal{C}}\right)$.

Proof. It suffices to consider the exact sequence $0 \rightarrow \mathcal{O}_{\mathbb{F}_{e}}(-\mathcal{C}) \rightarrow \mathcal{O}_{\mathbb{F}_{e}} \rightarrow \mathcal{O}_{\mathcal{C}} \rightarrow 0$ twisted by $L-M$ and to recall that $\operatorname{Ext}_{\mathbb{F}_{e}}^{1}(M, L) \simeq H^{1}\left(\mathbb{F}_{e}, L-M\right)$ and

$\operatorname{Ext}_{\mathcal{C}}^{1}\left(M_{\mid \mathcal{C}}, L_{\mid \mathcal{C}}\right) \simeq H^{1}\left(\mathcal{C},(L-M)_{\mid \mathcal{C}}\right)$.

We have the following

Theorem 3. Let $E_{\varepsilon}$ be a generic rank 2 vector bundle over $\mathbb{F}_{e}$ sitting in an exact sequence as follows:

$$
0 \rightarrow C_{0}+e f \rightarrow E_{\varepsilon} \rightarrow C_{0}+(e+\varepsilon) f \rightarrow 0 \quad\left(*_{\varepsilon}\right)
$$

with $e>0$ and $\varepsilon \in\{2,3\}$. Then $E$ is very ample.

Remark 3. Note that it is not possible that every $E_{\varepsilon}$ as above is very ample because, for instance, $E_{\varepsilon} \simeq\left(C_{0}+e f\right) \oplus\left(C_{0}+(e+\varepsilon) f\right)$ is obviously not ample.

Proof. (of Theorem 3) By Lemma 2 we have that $(s)_{0}$ is a set of $c_{2}\left(E_{\varepsilon}\right)$ distinct points for any smooth section $s$ of $E_{\varepsilon}$. To prove that $E_{\varepsilon}$ is very ample is equivalent to prove that the tautological divisor $T$ is very ample on $X$. To this aim we can use Proposition 3.1 of [A-B] and we prove that conditions $(i),(i i)$ and $(i i i)$ of that Proposition are satisfied.

(i) By using Proposition 3.5 of [A-B] (with $D=T, A=0, B=-Q$ ) it suffices to show that, for any effective divisor $Q$ of $\mathbb{F}_{e}$ such that $E_{\varepsilon}(-Q)$ is effective, we have (recall that $h^{0}\left(X, T-\pi^{*} Q\right)=h^{0}\left(\mathbb{F}_{e}, E_{\varepsilon}(-Q)\right)$ and so on):

$h^{0}\left(\mathbb{F}_{e}, E_{\varepsilon}(-Q)\right)<h^{0}\left(\mathbb{F}_{e}, E_{\varepsilon}\right)-2=2 e+2 \varepsilon+2$ and

$h^{0}\left(\mathbb{F}_{e}, Q\right)<h^{0}\left(\mathbb{F}_{e}, E_{\varepsilon}\right)-2=2 e+2 \varepsilon+2$.

Let $Q \sim q C_{0}+p f$ be an effective divisor of $\mathbb{F}_{e}$ such that $E_{\varepsilon}(-Q)$ is effective, obviously $q \leq 1$.

If $q=1$ and $p \geq 0$, from $\left(*_{\varepsilon}\right)$ we have that $E_{\varepsilon}(-Q)$ can be effective only if $0 \leq p \leq e+\varepsilon$ and we have to consider the following cases:

a) $p \in[0, e)$, then $h^{0}\left(\mathbb{F}_{e}, E_{\varepsilon}(-Q)\right)=2 e+2+\varepsilon-2 p<2 e+2 \varepsilon+2$;

$h^{0}\left(\mathbb{F}_{e}, Q\right)=p+1<2 e+2 \varepsilon+2$ as $p<e$.

b) $p=e$, then $h^{0}\left(\mathbb{F}_{e}, E_{\varepsilon}(-Q)\right)=2+\varepsilon<2 e+2 \varepsilon+2$;

$h^{0}\left(\mathbb{F}_{e}, Q\right)=e+2<2 e+2 \varepsilon+2$.

c) $p \in(e, e+\varepsilon]$, then $h^{0}\left(\mathbb{F}_{e}, E_{\varepsilon}(-Q)\right) \leq e+\varepsilon-p+1<2 e+2 \varepsilon+2$;

$h^{0}\left(\mathbb{F}_{e}, Q\right)=2 p+2-e<2 e+2 \varepsilon+2$ because $e>0$.

If $q=0$ and $p \geq 1$, from $\left(*_{\varepsilon}\right)$ we have that $E_{\varepsilon}(-Q)$ can be effective only if $1 \leq p \leq e+\varepsilon$. Here $h^{0}\left(\mathbb{F}_{e}, Q\right)=p+1 \leq e+\varepsilon+1<2 e+2 \varepsilon+2$, so we have to check only $h^{0}\left(\mathbb{F}_{e}, E_{\varepsilon}(-Q)\right)$ and we have to consider the following cases:

a) $p \in[1, e]$ and $\varepsilon-p \geq 0$, then $h^{0}\left(\mathbb{F}_{e}, E_{\varepsilon}(-Q)\right) \leq h^{0}\left(\mathbb{F}_{e}, C_{0}+(e-p) f\right)+$ $h^{0}\left(\mathbb{F}_{e}, C_{0}+(e+\varepsilon-p) f\right)=$

$=2 e+2 \varepsilon-3 p+3<2 e+2 \varepsilon+2$ as $p \geq 1$;

b) $p \in[1, e]$ and $\varepsilon-p<0$, then $h^{0}\left(\mathbb{F}_{e}, E_{\varepsilon}(-Q)\right) \leq h^{0}\left(\mathbb{F}_{e}, C_{0}+(e-p) f\right)+$ $h^{0}\left(\mathbb{F}_{e}, C_{0}+(e+\varepsilon-p) f\right)=$ 
$=2 e+\varepsilon-2 p+2<2 e+2 \varepsilon+2$;

c) $p \in(e, e+\varepsilon]$ and $\varepsilon-p \geq 0$ then $h^{0}\left(\mathbb{F}_{e}, E_{\varepsilon}(-Q)\right) \leq h^{0}\left(\mathbb{F}_{e}, C_{0}+(e+\varepsilon-p) f\right)=$ $e+2 \varepsilon-2 p+2<2 e+2 \varepsilon+2$

d) $p \in(e, e+\varepsilon]$ and $\varepsilon-p<0$ then $h^{0}\left(\mathbb{F}_{e}, E_{\varepsilon}(-Q)\right) \leq h^{0}\left(\mathbb{F}_{e}, C_{0}+(e+\varepsilon-p) f\right)=$ $e+\varepsilon-p+1<2 e+2 \varepsilon+2$.

(ii) For any smooth section $S \in|T|$, we have that the exact sequence

$0 \rightarrow \mathcal{O}_{X}(-S) \rightarrow \mathcal{O}_{X} \rightarrow \mathcal{O}_{S} \rightarrow 0$, twisted by $\mathcal{O}_{X}(T)$, proves that $H^{0}(X, T) \rightarrow$ $H^{0}\left(S, T_{\mid S}\right)$ is surjective because $h^{1}\left(X, \mathcal{O}_{X}\right)=0$.

(iii) As recalled in Lemma 2, any smooth element $S \in|T|$ is isomorphic to the blow up $\sigma$ of $\mathbb{F}_{e}$ at $\delta=c_{2}\left(E_{\varepsilon}\right)=e+\varepsilon$ distinct points $\left\{P_{1}, \ldots, P_{\delta}\right\}=(s)_{0}$ : the zero locus of the section $s \in H^{0}\left(\mathbb{F}_{e}, E_{\varepsilon}\right)$ corresponding to $S$ (see [B-S] Theorem 11.1.2); moreover $T_{\mid S} \sim \sigma^{*} c_{1}\left(E_{\varepsilon}\right)-F_{1}-\ldots-F_{\delta}=\sigma^{*}\left(2 C_{0}+(2 e+\varepsilon) f\right)-F_{1}-\ldots-F_{\delta}$ where $\left\{F_{i}\right\}$ are the exceptional divisors introduced by $\sigma$ (see Lemma 6.5 of $[\mathrm{A}-\mathrm{B}]$ and Lemma 2 of $[\mathrm{A}-\mathrm{T}]$ ). Hence, to prove the very ampleness of $T_{\mid S}$, we can use the Bese criterion (see Theorem 4.2 of [B]) with: $D \sim 2 C_{0}+(2 e+\varepsilon) f ; K_{\mathbb{F}_{e}} \sim-2 C_{0}-(2+e) f$; $\rho=h^{0}\left(\mathbb{F}_{e}, D-K_{\mathbb{F}_{e}}\right)-1 ; h=\rho-\frac{D^{2}-3 D K_{\mathbb{F}_{e}}+16}{2}$.

According to that criterion, we have to check that:

a) $D$ is very ample;

b) $\delta \leq\left[\frac{\rho-7}{3}\right]$, where [...] stands for the integer part;

c) $D^{2} \geq 10+4 h$

d) at most $C\left(D-K_{\mathbb{F}_{e}}-C\right)-3$ points among $\left\{P_{1}, \ldots, P_{\delta}\right\}$ can be contained in any curve $C \in\left|x C_{0}+y f\right|$ when $0 \leq x \leq 4$ and $0 \leq y \leq\left[\frac{3 e+2+\varepsilon}{2}\right]$, with $(x, y) \neq(0,0)$.

Let us examine the four items.

a) It is well known that a divisor $q C_{0}+p f$ on a surface $\mathbb{F}_{e}$ is very ample if $q>0$ and $p>q e$.

b) It suffices to show that $\delta=e+\varepsilon \leq \frac{\rho-7}{3}-1=\frac{\rho-10}{3}$. Here $D-K_{\mathbb{F}_{e}} \sim$ $4 C_{0}+(3 e+2+\varepsilon)$ and $\rho=h^{0}\left(\mathbb{P}^{1}, \mathcal{O}_{\mathbb{P}^{1}}(2+\varepsilon-e)\right)+6 e+4 \varepsilon+11$; hence we have to check: $3 e+3 \varepsilon \leq h^{0}\left(\mathbb{P}^{1}, \mathcal{O}_{\mathbb{P}^{1}}(2+\varepsilon-e)\right)+6 e+1+4 \varepsilon$ and this is true.

c) By using the value of $h$, the inequality can be rewritten as $3 D^{2}+22-6 D K_{\mathbb{F}_{e}} \geq$ $4 \rho$ and we have to check:

$3(-4 e+8 e+4 \varepsilon)+22+6(2 e+2 \varepsilon+4) \geq 4\left[h^{0}\left(\mathbb{P}^{1}, \mathcal{O}_{\mathbb{P}^{1}}(2+\varepsilon-e)\right)+6 e+4 \varepsilon+11\right]$, i.e. $8 \varepsilon+2 \geq 4\left[h^{0}\left(\mathbb{P}^{1}, \mathcal{O}_{\mathbb{P}^{1}}(2+\varepsilon-e)\right)\right]$, and this is true because $8 \varepsilon+2 \geq 4(3+\varepsilon-e) \geq$ $4\left[h^{0}\left(\mathbb{P}^{1}, \mathcal{O}_{\mathbb{P}^{1}}(2+\varepsilon-e)\right)\right]$ when $\varepsilon=3$ and also when $\varepsilon=2$ because $e>0$.

d) The condition is obviously true if $C\left(D-K_{\mathbb{F}_{e}}-C\right)-3=x^{2} e-x e+(2+$ $\varepsilon) x+4 y-2 x y-3>\delta=e+\varepsilon$ and it is easy to see that this is true when $x \in[2,4]$. For the other values of $x$ let us remark that $(s)_{0}$ is a set of points lying on a smooth rational curve $\Gamma_{s} \in\left|C_{0}+(e+\varepsilon) f\right|$. In fact $s$ is not a generic section, however we can use Proposition 2 iii): in our situation $V$ is the linear space of sections giving rise to smooth surfaces $S \in|T|$ satisfying two linear conditions (see [A-B] Propositions 3.1 and 3.5), hence $V$ has codimension 2 at most, hence $\operatorname{dim}\left[\operatorname{Im}\left(V \rightarrow H^{0}\left(\mathbb{F}_{e}, M\right)\right)\right] \geq h^{0}\left(\mathbb{F}_{e}, C_{0}+(e+\varepsilon) f\right)-2=e+2 \varepsilon>e+\varepsilon+1$.

If $x=0$ (hence $y \geq 1) C\left(D-K_{\mathbb{F}_{e}}-C\right)-3=4 y-3$ and every $C$ is the union of $y$ fibres of $\mathbb{F}_{e}$. As the $\delta$ points belong to a smooth section $\Gamma_{s} \in\left|C_{0}+(e+\varepsilon) f\right|$ of $\mathbb{F}_{e}$, no fibre may contain more than one point. Therefore the maximal number of points among $\left\{P_{1}, \ldots, P_{\delta}\right\}$ belonging to a curve $C \in|y f|$ is bounded by $y$. The condition is satisfied because $y \leq 4 y-3$. 
If $x=1, C\left(D-K_{\mathbb{F}_{e}}-C\right)-3=2 y+\varepsilon-1$. As above, the $\delta$ points belong to a smooth section $\Gamma_{s}$. Let us call $\theta$ the maximal number of points among $\left\{P_{1}, \ldots, P_{\delta}\right\}$ belonging to a curve $C \in\left|C_{0}+y f\right|$; obviously $\theta \leq C \Gamma_{s}=y+\varepsilon \leq 2 y+\varepsilon-1$ unless $y=0$. If $y=0$ then $C=C_{0}$ and $\theta=\sharp\left((s)_{0} \cap C_{0}\right)$; we need to prove that $\sharp\left((s)_{0} \cap C_{0}\right) \leq \varepsilon-1$. Let us consider the restriction of $\left(*_{\varepsilon}\right)$ to $C_{0}$, we get $0 \rightarrow \mathcal{O}_{\mathbb{P}^{1}} \rightarrow E_{\varepsilon \mid C_{0}} \rightarrow \mathcal{O}_{\mathbb{P}^{1}}(\varepsilon) \rightarrow 0$. As $E_{\varepsilon}$ is generic, then $E_{\varepsilon \mid C_{0}} \neq \mathcal{O}_{\mathbb{P}^{1}} \oplus \mathcal{O}_{\mathbb{P}^{1}}(\varepsilon)$ by Lemma 3 , hence $E_{\varepsilon \mid C_{0}} \simeq \mathcal{O}_{\mathbb{P}^{1}}(a) \oplus \mathcal{O}_{\mathbb{P}^{1}}(b)$ with $0<a \leq b \leq \varepsilon-1$. By Lemma 2 we have $\sharp\left((s)_{0} \cap C_{0}\right) \leq \varepsilon-1$.

Corollary 3. Let $E_{\varepsilon}$ be any rank 2 vector bundle over $\mathbb{F}_{e}$ sitting in an exact sequence as follows:

$$
0 \rightarrow C_{0}+e f \rightarrow E_{\varepsilon} \rightarrow C_{0}+(e+\varepsilon) f \rightarrow 0 \quad\left(*_{\varepsilon}\right)
$$

with $e>0$ and $\varepsilon \in\{2,3\}$. Assume that at least one of the following assumptions is satisfied:

I) for any section $s \in H^{0}\left(\mathbb{F}_{e}, E_{\varepsilon}\right)$, corresponding to a smooth surface $S \in|T|$, $\sharp\left((s)_{0} \cap C_{0}\right) \leq \varepsilon-1$;

II) for any section $s \in H^{0}\left(\mathbb{F}_{e}, E_{\varepsilon}\right)$, corresponding to a smooth surface $S \in|T|$, $(s)_{0}$ is a set of distinct points in general position according to Definition 1;

III) $E_{\varepsilon \mid C_{0}}$ is ample;

then $E_{\varepsilon}$ is very ample.

Proof. I) By looking at the proof of Theorem 3 we see that we have used the assumption that $E_{\varepsilon}$ is generic only to prove that $\sharp\left((s)_{0} \cap C_{0}\right) \leq \varepsilon-1$ for sections $s \in H^{0}\left(\mathbb{F}_{e}, E_{\varepsilon}\right)$, corresponding to smooth surfaces $S \in|T|$.

$I I)$ Definition 1 implies that $(s)_{0} \cap C_{0}=\emptyset$, hence $\sharp\left((s)_{0} \cap C_{0}\right) \leq \varepsilon-1$ and we can proceed as in $I$ ).

$I I I)$ If $E_{\varepsilon \mid C_{0}}$ is ample, $E_{\varepsilon \mid C_{0}} \neq \mathcal{O}_{\mathbb{P}^{1}} \oplus \mathcal{O}_{\mathbb{P}^{1}}(\varepsilon)$ and we can argue as at the end of the proof of Theorem 3 to get $\sharp\left((s)_{0} \cap C_{0}\right) \leq \varepsilon-1$, then we can proceed as in $\left.I\right)$.

\section{VERY AMPLENESS AND GENERAL POSITION, OTHER EXAMPLES}

Up to now we have always supposed that $L \sim C_{0}+\alpha f$ in any sequence $(\epsilon)$. It is natural to ask whether Definition 1 is useful to prove the very ampleness of vector bundles $E$ when $L \sim q C_{0}+\alpha f$ with $q \geq 2$, and $\alpha \geq e$ to assure that the generic element of $|L|$ is a smooth curve. In these cases $h^{1}\left(\mathbb{F}_{e}, L\right)=0$ is not necessarily true, hence it is not necessarily true that $(s)_{0}$ belongs to a smooth rational curve $\Gamma_{s} \in|M|$ for generic sections $s$ of $E$. However, by using Proposition 2, iii) it is possible to prove, with a long calculation omitted here, that this is true if $q=2$ and in very few other cases. This fact explains why, in this section, we will consider only the case $L \sim 2 C_{0}+\alpha f$; we will see that this generalization does not produce different results. As usual, we also assume that $\alpha \leq e+\beta-2$, i.e. $H^{1}\left(\mathbb{F}_{e}, L-M\right) \neq 0$, to assure that $(\epsilon)$ with $Z=\emptyset$ is not necessarily splitting. Let us start with a Proposition analogous to Proposition 5:

Proposition 6. Let $E$ be a very ample rank 2 vector bundle over $\mathbb{F}_{e}$ sitting in an exact sequence as follows:

$$
0 \rightarrow 2 C_{0}+\alpha f \rightarrow E \rightarrow C_{0}+\beta f \rightarrow 0
$$

and let $\Delta:=(s)_{0}$ be the zero locus of a generic section $s$ of $E$. Assume that $\delta:=$ length $(\Delta) \geq 1$ and $0<e \leq \alpha \leq e+\beta-2$ and let $\chi$ be any non negative integer. Then $\Delta$ can be in general position only if one of the following cases holds: 
i) $e \geq 2, \alpha=e+1, \beta=2 e+\chi, \delta=3 e+1+2 \chi$;

and in this case $\Delta$ is in fact in general position if and only if $\Delta \cap C_{0}=\emptyset$.

ii) $e=1, \alpha=2, \beta \geq 3, \delta=2 \beta$;

and in this case $\Delta$ is in fact in general position if and only if $\Delta \cap C_{0}=\emptyset$.

iii) $e \geq 1, \alpha=e, \beta=2 e+1+\chi, \delta=3 e+2+2 \chi$;

and in this case $\Delta$ is in fact in general position if and only if $\Delta \cap C_{0}=\emptyset$.

Proof. As $E$ is very ample then $C_{0}+\beta f$ must be ample, hence $\beta>e ; c_{1}(E)$ must be ample, hence $\alpha+\beta>3 e$; moreover $\Delta$ is given by a set of $\delta=\alpha+2(\beta-e)$ distinct points, belonging to a smooth rational curve $\Gamma_{s}$ by Proposition 2 ii) and iii). In fact we have to check that $\zeta:=\operatorname{dim}\left[\operatorname{Im}\left(H^{0}\left(\mathbb{F}_{e}, L\right) \rightarrow H^{0}\left(\mathbb{F}_{e}, L\right)\right)\right]>\beta+1$; as $\zeta \geq h^{0}\left(\mathbb{F}_{e}, M\right)-h^{1}\left(\mathbb{F}_{e}, L\right)=2 \beta+2-e-h^{1}\left(\mathbb{F}_{e}, L\right)$ it suffices to check that $\beta \geq e+h^{1}\left(\mathbb{F}_{e}, L\right)=e+h^{1}\left(\mathbb{F}_{e}, 2 C_{0}+\alpha f\right)$. If $\alpha>2 e-2$ this is true because $h^{1}\left(\mathbb{F}_{e}, L\right)=0$. If $e \leq \alpha \leq 2 e-2$, this is true because $h^{1}\left(\mathbb{F}_{e}, L\right)=2 e-\alpha-1$ and $\alpha+\beta>3 e$.

Therefore, as in Proposition 5, we can use Corollary 2 to decide when $\Delta$ can be in general position, always assuming that $\Delta \cap C_{0}=\emptyset$, (i.e. that $\varphi_{\Delta}(0)$ is the expected value 0 ).

Of course case $a$ ) is not possible. In case $b$ ) it would be $\alpha \leq 2 e-1,1 \leq \delta=$ $2+\alpha \leq e+3$ and $\alpha+e+1>3 e$, but this is not possible because $\alpha \geq e$. In case c) it would be $1 \leq \delta=4+\alpha \leq e+2$ and this is not possible because $\alpha \geq e$. In case $d$ ) it would be: $\alpha \leq 2 e ; e+3 \leq \delta=4+\alpha \leq e+5 ; \alpha+2>2 e$; as $\alpha \geq e$ this is possible only if $(e, \alpha, \beta) \in\{(1,1,3),(1,2,3),(2,3,4)\}$. In case $e)$ it would be $\delta=\alpha+2(\beta-e) \leq \beta$ with $\beta=e+3+\eta, \eta \geq 0$ and this is not possible because $\alpha \geq e$. In case $f)$ it would be $\delta=\alpha+2(\beta-e)<2 \beta-1-e$ and this is not possible because $\alpha \geq e$.

In case $g$ ) it would be $e+\delta=e+\alpha+2(\beta-e) \leq 2 \beta+1$ with $\beta=e+3+\eta, \eta \geq 0$. This is possible only if $\alpha \in\{e, e+1\}$ because $\alpha \geq e$. If $\alpha=e+1$ the condition $\alpha+\beta>3 e$ implies $\eta>e-4$, hence $\beta=2 e+\chi$. If $\alpha=e$ the same condition implies $\eta>e-3$, hence $\beta=2 e+1+\chi$.

If $\alpha=e+1$ we get $i$ ), enclosing $(e, \alpha, \beta)=(2,3,4)$ of case $d$ ), and we get $i i)$, enclosing $(e, \alpha, \beta)=(1,2,3)$ of case $d)$. If $\alpha=e$ we get iii) enclosing $(e, \alpha, \beta)=$ $(1,1,3)$ of case $d)$.

Now we can prove the following:

Theorem 4. Let $E$ be any rank 2 vector bundle over over $\mathbb{F}_{e}$ sitting in an exact sequence as follows:

$$
0 \rightarrow 2 C_{0}+\alpha f \rightarrow E \rightarrow C_{0}+\beta f \rightarrow 0 .
$$

Let us define $\mu:=\max \left\{\sharp\left((s)_{0} \cap C_{0}\right) \mid s \in H^{0}\left(\mathbb{F}_{e}, E\right)\right.$ corresponding to a smooth surface $S \in|T|\}$ and let $\chi$ be any non negative integer. If at least one of the following cases holds:

i) $e \geq 2, \alpha=e+1, \beta=2 e+\chi, \delta=3 e+1+2 \chi, \mu \leq-3 e+\alpha+\beta-1=\chi$;

ii) $e=1, \alpha=2, \beta \geq 3, \delta=2 \beta$ and $E$ is generic;

iii) $e \geq 1, \alpha=e, \beta=2 e+1+\chi, \delta=3 e+2+2 \chi, \mu \leq-3 e+\alpha+\beta-1=\chi$; then $E$ is very ample.

Remark 4. Note that, as for Theorem 3, it is not possible that every $E$ in case ii) is very ample because, for instance, $E \simeq\left(2 C_{0}+2 f\right) \oplus\left(C_{0}+\beta f\right)$ is obviously not ample. 
Proof. (of Theorem 4) We proceed as in the proof of Theorem 3 and to prove that $E$ is very ample we use again Proposition 3.1 of [A-B] and we prove that conditions (i), (ii) and (iii) of that Proposition are satisfied for every case $i$ ), ii), iii).

(i) By using Proposition 3.5 of [A-B] (with $D=T, A=0, B=-Q$ ) it suffices to show that, for any effective divisor $Q \sim \xi C_{0}+\eta f$ of $\mathbb{F}_{e}$ such that $E(-Q)$ is effective, we have:

$h^{0}\left(\mathbb{F}_{e}, E(-Q)\right)<h^{0}\left(\mathbb{F}_{e}, E\right)-2$ and

$h^{0}\left(\mathbb{F}_{e}, Q\right)<h^{0}\left(\mathbb{F}_{e}, E\right)-2$.

Note that $h^{0}\left(\mathbb{F}_{e}, E\right)-2 \geq h^{0}\left(\mathbb{F}_{e}, 2 C_{0}+\alpha f\right)+h^{0}\left(\mathbb{F}_{e}, C_{0}+\beta f\right)-h^{1}\left(\mathbb{F}_{e}, 2 C_{0}+\alpha f\right)=$ $3 e+2 \chi+6$ in case $i) ; h^{0}\left(\mathbb{F}_{1}, E\right)-2 \geq 2 \beta+5$ in case $\left.i i\right) ; h^{0}\left(\mathbb{F}_{e}, E\right)-2 \geq 3 e+2 \chi+5$ in case $i$ ii).

Obviously $E(-Q)$ can be effective only if $0 \leq \xi \leq 2$.

Let us assume $\xi=2 ; E(-Q)$ is effective only if $\alpha \geq \eta$.

We have that $h^{0}\left(\mathbb{F}_{e}, E(-Q)\right) \leq \alpha-\eta+1<h^{0}\left(\mathbb{F}_{e}, E\right)-2$ in any case.

Concerning $h^{0}\left(\mathbb{F}_{e}, Q\right)$ we have:

in case $i): h^{0}\left(\mathbb{F}_{e}, Q\right) \leq e+4<3 e+2 \chi+6 \leq h^{0}\left(\mathbb{F}_{e}, E\right)-2$;

in case $i$ i) : $h^{0}\left(\mathbb{F}_{1}, Q\right) \leq 5<2 \beta+5 \leq h^{0}\left(\mathbb{F}_{1}, E\right)-2$;

in case $i i i): h^{0}\left(\mathbb{F}_{e}, Q\right) \leq e+2<3 e+2 \chi+5 \leq h^{0}\left(\mathbb{F}_{e}, E\right)-2$.

Let us assume $\xi=1 ; E(-Q)$ is effective only if $\beta \geq \eta$. We have:

in case $i): h^{0}\left(\mathbb{F}_{e}, E(-Q)\right) \leq e+4+\beta+1=3 e+\chi+4<3 e+2 \chi+6 \leq h^{0}\left(\mathbb{F}_{e}, E\right)-2$; in case $i$ i : $h^{0}\left(\mathbb{F}_{1}, E(-Q)\right) \leq 5+\beta+1<2 \beta+5 \leq h^{0}\left(\mathbb{F}_{1}, E\right)-2$;

in case iii) : $h^{0}\left(\mathbb{F}_{e}, E(-Q)\right) \leq e+2+\beta+1=3 e+\chi+3<3 e+2 \chi+5 \leq$ $h^{0}\left(\mathbb{F}_{e}, E\right)-2$.

Concerning $h^{0}\left(\mathbb{F}_{e}, Q\right)$ we have:

in case $i): h^{0}\left(\mathbb{F}_{e}, Q\right) \leq 2 \beta-e+2=3 e+2 \chi+2<3 e+2 \chi+6 \leq h^{0}\left(\mathbb{F}_{e}, E\right)-2$; in case $i$ i) : $h^{0}\left(\mathbb{F}_{1}, Q\right) \leq 2 \beta+1<2 \beta+5 \leq h^{0}\left(\mathbb{F}_{1}, E\right)-2$;

in case $i i i): h^{0}\left(\mathbb{F}_{e}, Q\right) \leq 2 \beta-e+2=3 e+2 \chi+4<3 e+2 \chi+5 \leq h^{0}\left(\mathbb{F}_{e}, E\right)-2$.

Let us assume $\xi=0 ; E(-Q)$ is effective only if $\beta \geq \eta$ (and $\eta \geq 1$ ), hence $h^{0}\left(\mathbb{F}_{e}, Q\right)=\eta+1 \leq \beta+1<h^{0}\left(\mathbb{F}_{e}, E\right)-2$ in any case. Concerning $h^{0}\left(\mathbb{F}_{e}, E(-Q)\right)$ we argue in a different way. Firstly let us remark that $h^{0}\left(\mathbb{F}_{e}, E(-Q)\right) \leq h^{0}\left(\mathbb{F}_{e}, E(-f)\right)=$ $h^{0}\left(X, T-\pi^{*} f\right)$. Secondly, let us remark that $E_{\mid f}$ is very ample and that the ruled surface $\mathbb{P}\left(E_{\mid f}\right)$ is a cubic scroll in $\mathbb{P}^{4}$, hence $h^{0}\left(X, T-\pi^{*} f\right)=h^{0}(X, T)-1-4$ (as $h^{0}(X, T)=h^{0}\left(\mathbb{F}_{e}, E\right) \geq 5$ in any case), therefore $h^{0}\left(\mathbb{F}_{e}, E(-Q)\right) \leq h^{0}(X, T)-4<$ $h^{0}\left(\mathbb{F}_{e}, E\right)-2$.

(ii) Let $X$ be $\mathbb{P}(E)$ as usual. For any smooth section $S \in|T|$, we have that the exact sequence $0 \rightarrow \mathcal{O}_{X}(-S) \rightarrow \mathcal{O}_{X} \rightarrow \mathcal{O}_{S} \rightarrow 0$, twisted by $\mathcal{O}_{X}(T)$, proves that $H^{0}(X, T) \rightarrow H^{0}\left(S, T_{\mid S}\right)$ is surjective because $h^{1}\left(X, \mathcal{O}_{X}\right)=0$.

(iii) As in the proof of Theorem 3 we use the Bese criterion (see Theorem 4.2 of [B]) with: $D \sim 3 C_{0}+(\alpha+\beta) f ; K_{\mathbb{F}_{e}} \sim-2 C_{0}-(2+e) f ; \rho=h^{0}\left(\mathbb{F}_{e}, D-K_{\mathbb{F}_{e}}\right)-1$; $h=\rho-\frac{D^{2}-3 D K_{\mathbb{F}_{2}}+16}{2}=0 ; \delta=-2 e+2 \beta+\alpha$.

According to that criterion, we have to check that:

a) $D$ is very ample;

b) $-2 e+2 \beta+\alpha \leq\left[\frac{\rho-7}{3}\right]$, where [...] stands for the integer part;

c) $D^{2} \geq 10+4 h$

d) at most $C\left(D-K_{\mathbb{F}_{e}}-C\right)-3$ points among $\left\{P_{1}, \ldots, P_{\delta}\right\}$ can be contained in any curve $C \in\left|x C_{0}+y f\right|$ when $0 \leq x \leq 1$ and $0 \leq y \leq\left[\frac{\alpha+\beta+2+e}{2}\right]$, with $(x, y) \neq(0,0)$. 
Let us examine the four items. $a$ ) is obviously true because $\alpha+\beta>3 e$ in any case. Let us consider $b$ ).

In case $i$ ) we have $\rho=9 e+6 \chi+23$ if $3+\chi \geq e$ and $\rho=10 e+5 \chi+19$ if $3+\chi<e$, while $\delta=3 e+2 \chi+1$, hence $b$ ) is satisfied.

In case $i$ ) we have $\rho=6 \beta+20$ and $\delta=2 \beta \leq\left[\frac{6 \beta+13}{3}\right]$, hence $b$ ) is satisfied.

In case $i$ ii) we have the same values for $\rho$ as in case $i$ ) and $\delta=3 e+2 \chi+2$, hence $b)$ is satisfied in this case too.

Let us consider $c)$. We have $h=\rho-(6 \alpha+6 \beta-9 e+17)$ and $D^{2}-10=$ $6 \alpha+6 \beta-9 e-10$. In cases $i$ ) and $i i i), h=0$ if $3+\chi \geq e$ and $h=e-\chi-4$ if $3+\chi<e$, in case $i i) h=0$. Hence $c$ ) is satisfied in any case.

Let us consider $d$ ). Let us recall that $\Delta$ is a set of distinct points belonging to a smooth section $\Gamma_{s} \in\left|C_{0}+\beta f\right|$. In fact we can use Proposition 2 iii) as in the proof of Theorem 3: in our situation $V$ has codimension 2 at most and we have to check that $\zeta:=\operatorname{dim}\left[\operatorname{Im}\left(V \rightarrow H^{0}\left(\mathbb{F}_{e}, M\right)\right)\right]>\beta+1$ in every case. By recalling that $\zeta \geq h^{0}\left(\mathbb{F}_{e}, C_{0}+\beta f\right)-h^{1}\left(\mathbb{F}_{e}, 2 C_{0}+\alpha f\right)-2$, in case $\left.i\right)$ we have: $\zeta \geq 2 \beta+4-2 e-2>\beta+1$ if $\beta+1>2 e$ and this is true; in case $i i)$ we have: $\zeta \geq 2 \beta-1>\beta+1$; in case iii) we have: $\zeta \geq 2 \beta+3-2 e-2>\beta+1$, if $\beta>2 e$ and this is true.

If $x=0$ (hence $y \geq 1) C\left(D-K_{\mathbb{F}_{e}}-C\right)-3=5 y-3$ and every $C$ is the union of $y$ fibres of $\mathbb{F}_{e}$. As the $\delta$ points belong to a smooth section $\Gamma_{s} \in\left|C_{0}+\beta f\right|$ of $\mathbb{F}_{e}$, no fibre may contain more than one point. Therefore the maximal number of points among $\left\{P_{1}, \ldots, P_{\delta}\right\}$ belonging to a curve $C \in|y f|$ is bounded by $y$. The condition is satisfied in any case because $y \leq 5 y-3$.

If $x=1, C\left(D-K_{\mathbb{F}_{e}}-C\right)-3=3(y-e)+\alpha+\beta-1$ and, as we have shown above, the $\delta$ points belong to a smooth section $\Gamma_{s}$. Let us call $\theta$ the maximal number of points among $\left\{P_{1}, \ldots, P_{\delta}\right\}$ belonging to a curve $C \in\left|C_{0}+y f\right|$.

If $y=0$ then $C=C_{0}$ and $\theta=\sharp\left((s)_{0} \cap C_{0}\right) \leq \mu$; we need to prove that $\mu \leq$ $-3 e+\alpha+\beta-1$. This is true by assumption in cases $i$ ) and $i i i)$. In case $i i)$ we can argue as in the proof of Theorem 3: the restriction of $E$ to $C_{0}$ gives the following non splitted exact sequence (non splitted because $E$ is generic, see Lemma 3): $0 \rightarrow \mathcal{O}_{\mathbb{P}^{1}} \rightarrow E_{\mid C_{0}} \rightarrow \mathcal{O}_{\mathbb{P}^{1}}(\beta-1) \rightarrow 0$ hence, by Lemma 2 , we have that $\mu \leq \beta-2 \leq-3 e+\alpha+\beta-1$.

If $1 \leq y<e, C$ is always the union of $C_{0}$ and $y$ fibres of $\mathbb{F}_{e}$; as $\Gamma_{s}$ is a smooth section, the maximal number of points of $\Delta$ belonging to $C$ is less than or equal to $y+\mu$. Then it suffices that $y+\mu \leq 3(y-e)+\alpha+\beta-1$ and this is true in any case.

If $y \geq e$, obviously $\theta \leq C \Gamma_{s}=-e+\beta+y \leq 3(y-e)+\alpha+\beta-1$ in any case.

As for Theorem 3 we have the following Corollary to Theorem 4:

Corollary 4 . Let $E$ be any rank 2 vector bundle over $\mathbb{F}_{1}$ sitting in an exact sequence as follows:

$$
0 \rightarrow 2 C_{0}+2 f \rightarrow E \rightarrow C_{0}+\beta f \rightarrow 0 .
$$

with $\beta \geq 3$. Assume that at least one of the following assumptions holds:

I) for any section $s \in H^{0}\left(\mathbb{F}_{1}, E\right)$, corresponding to a smooth surface $S \in|T|$, $\sharp\left((s)_{0} \cap C_{0}\right) \leq \beta-2$;

$I I)$ for any section $s \in H^{0}\left(\mathbb{F}_{1}, E\right)$, corresponding to a smooth surface $S \in|T|$, $(s)_{0}$ is a set of points in general position according to Definition 1;

II) $E_{\mid C_{0}}$ is ample; then $E$ is very ample. 
Proof. Exactly as the proof of Corollary 3.

Some final remarks. Vector bundles considered by Theorem 4 are very popular because their splitting type is $(2,1)$ for any fibre, hence they are uniform, see for instance the introduction of [F-F]. It is natural to ask whether the very ampleness of vector bundles $E$ analyzed in [F-F] can be proved by using the general position of $(s)_{0}$ at least when $s$ is a section of $E$ giving rise to a smooth surface $S \in|T|$. The answer is not. In fact, when a very ample vector bundle sits in an exact sequence as

$$
0 \rightarrow L \rightarrow E \rightarrow C_{0}+\beta f \rightarrow 0
$$

on a surface $\mathbb{F}_{e}$ (hence $\beta>e$ ) we know that $(s)_{0} \subset \Gamma_{s} \in\left|C_{0}+\beta f\right|$, therefore, if we want that $(s)_{0}$ is in general position, it must be $h^{0}\left(\mathbb{F}_{e}, C_{0}+\beta f\right)=2 \beta+2-e>c_{2}(E)$. In [F-F] the authors assume $\beta=c_{2}(E)-b+2 e$ (see (3.2)) and $c_{2}(E)<2 b-4 e$ (see Remark 4.2) with $e \geq 2$, hence $2 \beta+2-e \leq c_{2}(E)$.

Acknowledgements: we wish to thank A. Lanteri for fruitful conversations about our application of Theorem 11.1.2 of [B-S].

\section{REFERENCES}

[A-B] A.Alzati-G.M.Besana: "Criteria for very ampleness of rank two vector bundles over ruled surfaces". Can. J. Math. 62 (6) (2010) pp. 1201-1227.

[A-T] A.Alzati-A.Tortora: "Rank 2 vector bundle over $\mathbb{P}^{2}(\mathbb{C})$ whose sections have special properties". Rev. Mat. Complut. 28 (3) (2015) pp.623-654.

[B-C] L.Bazzotti-M.Casanellas: "Separators of points on algebraic surfaces". J. Pure Appl. Algebra 207 (2006) pp. 316-326.

[B-S] M.C.Beltrametti-A.J.Sommese:" The adjunction theory of complex projective varieties" de Gruyter Expositions in Mathematics 16, Walter de Gruyter \& Co., Berlin 1995.

[B-B] G.M.Besana-A.Biancofiore: "Degree eleven projective manifolds of dimension greater than or equal to three". Forum Math. 17 (5) (2005) pp. 711-733.

[B] E.Bese: "On the Spannedness and Very Ampleness of Certain Line Bundles on the Blowups of $\mathbb{P}_{\mathbb{C}}^{2}$ and $\mathbb{F}_{r}$ ". Math. Ann. 262 (1983) pp. 225-238.

[F] R.Friedman: "Algebraic surfaces and Holomorphic Vector Bundles". Universitexts, Springer-Verlag, New York, 1998.

[F-F] L.Fania-F.Flamini: "Hilbert schemes of some threefold scrolls over $\mathbb{F}_{e}$ ". Adv. Geom. 16 (4) (2016) pp. 413-436.

[F-L1] L.Fania-E.L.Livorni: "Degree nine manifolds of dimension greater than or equal to 3". Math. Nachr. 169 (1994) pp. 117-134.

[F-L2] L.Fania-E.L.Livorni: "Degree ten manifolds of dimension n greater than or equal to 3". Math. Nachr. 188 (1997) 79-108.

[G-H] P.Griffiths-J.Harris: "Principles of Algebraic Geometry" Wiley Interscience Publication, New York, 1994.

[I1] P.Ionescu: "Embedded projective varieties of small invariants" in Proceedings of the Week of Algebraic Geometry, Bucharest 1982, Springer Lecture Notes in Mathematics 1056 (1984) pp. 142-186.

[I2] P.Ionescu: "Embedded projective varieties of small invariants III" in Algebraic Geometry, L'Aquila 1988, Springer Lecture Notes in Mathematics 1417 (1990) pp. 138-154.

Dipartimento di Matematica Univ. di Milano, via C. Saldini 50 20133-Milano (Italy)

E-mail address: alberto.alzati@unimi.it

Dipartimento di Matematica Univ. di Milano, via C. Saldini 50 20133-Milano (Italy)

E-mail address: alfonso.tortora@unimi.it 